$\mathrm{IgE}$ is $268 \mathrm{IU} / \mathrm{ml}$. Specific IgE to Tomato- 0.02, Strawberry0.01 , Kiwi fruit- 0.03, Peanut- 1.25, Hazelnut-0.97, Orange0.87, Latex-1.18,Birch pollen-1.15. Thyroid function test, MRI brain were normal. Investigations performed for Wilson's disease, which were unremarkable. Coeliac screen was normal. Hereditary angioedema scree is normal There was no record of basal serum tryptase level.

Treatment Initial diagnosis of idiopathic anaphylaxis made. Later with new neurological and eye symptoms, diagnosis of mast cell activation syndrome suspected, and he was started on trial of oral sodium cromoglycate capsules.

In next clinic follow up, his symptoms were completely resolved and feedback from school was excellent in terms of concentration.

Discussion Symptoms of allergic disorders are very wide. Mast cell activation syndrome especially with multisystem involvement should be a differential to Idiopathic Anaphylaxis.

\section{CLINICAL, IMMUNOLOGICAL AND GENETIC FINDINGS IN 22 PATIENTS WITH COMBINED IMMUNODEFICIENCY TREATED IN A SPECIALIZED CENTER IN CROATIA}

Nevenka Cigrovski* ${ }^{*}$ Darko Richter, Dorian Tješić-Drinković, Ivan Bambir, Drago Batinić, Antonija Babić, Lejla Kurić, Ines Bojanić, Zorana Grubić, Goran Tešović, Maja Vrdoljak, Jadranka Kelečić. University Hospital Centre Zagreb, Department of Pediatrics, Division of Respiratory Diseases, Clinical Immunology and Allergology

\subsection{6/archdischild-2021-europaediatrics.155}

To gain insight into the peculiarities of clinical presentation and time of diagnosis in the population of patients with combined immunodeficiency treated in our center.

We retrospectively analyzed 22 patients with combined immunodeficiency treated in our center in the past 25 years. Clinical findings were collected, including age of presentation and age of diagnosis, genetic defects, consanguinity, family history, number and site of infections, presence of diarrhea, failure to thrive, skin rash, vaccine complications and type of treatment. Immunological findings included WBC with absolute counts, lymphocyte subsets and immunoglobulin levels.

Patients $(n=22)$ with severe combined immunodeficiency $(n=17)$ and other combined immunodeficiencies $(n=5)$ were included in the study. Sixteen patients (77\%) were male. The most common clinical manifestations were opportunistic infections. Most of the patients were diagnosed after 3 months of age. Prenatal diagnosis was performed in one patient $(4.5 \%)$, two patients

(9\%) did not undergo genetic analysis. The spectrum of genetic defect in our cohort show wide heterogeneity. Seventeen patients $(77 \%)$ underwent HSCT, one patient $(4.5 \%)$ is on enzyme replacement therapy. None of our patients have undergone gene therapy. Twelve patients $(54.5 \%)$ died, of which four (18\%) died before HSCT.

Combined immunodeficiencies are fatal without definitive therapy (HSCT or gene therapy). The outcomes are better if infants are diagnosed and treated in pre-symptomatic stage, preferably before 3.5 months of age. More and more countries are implementing newborn screening for primary immunodeficiency diseases in their health care systems to diagnose patients as early as possible and to prevent infections. For the same reason, we advocate for expanding our national neonatal screening program

\section{OMALIZUMAB FOR CHRONIC URTICARIA IN A PATIENT YOUNGER THAN 12 YEARS - CASE REPORT}

Marija Pečnjak*, Blaženka Kljajić-Bukvić, Mario Blekić, Mirjana Turkalj. General Hospital 'DR. Josip Benčević,', Slavonski Brod

\subsection{6/archdischild-2021-europaediatrics. 156}

Urticaria is common condition in children and most cases are acute and self-limiting. Chronic urticaria in children is rare and defined with daily/almost daily presence of symptoms lasting for at least six weeks. It has two forms, chronic spontaneous urticaria (CSU) and chronic inducible urticaria.

CSU is usually considered as a disease of unknown origin but in some children an underlining autoimmune mechanism or atopic disease may be present.

Current treatment guidelines recommend elimination of revealed trigger factors, second generation $\mathrm{H} 1$-antihistamines and in unresponsive patients, ciclosporin or omalizumab (anti$\operatorname{IgE}$ antibody).

Data about effectiveness and safety of omalizumab treatment in paediatric population are limited.

We present a 9-year-old girl with CSU who was successfully treated with omalizumab.

A 9-year-old girl presented with urticaria and angioedema of the eyelids lasting for 4 months. She was treated with H1antihistamines without success. Her mother reported sun exposure, exercise, egg and milk consumption as trigger factors. Laboratory investigations including inflammatory markers, complete blood count, general biochemistry, immunoglobulin levels and protein electrophoresis were normal. Total IgE level was $15.9 \mathrm{kIU} / \mathrm{L}$ and specific IgE for milk and egg was negative. Thyroid function tests were pathologic, antithyroid antibodies were increased but not requiring substitute levothyroxine therapy. Thyroid ultrasound showed diffusely enlarged thyroid gland with a heterogeneous echotexture. She also had homogenous pattern of antinuclear antibodies on indirect immunofluorescence without other symptoms/signs of a rheumatologic disease.

Initial weekly Urticaria Activity Score (UAS7) was 28-30. Therapy with $\mathrm{H} 1$-antihistamines was continued with the fourfold increase in dosage without success. She also received systemic corticosteroids in acute flares. Further investigations revealed negative autologous serum skin test and positive basophil activation test. Due to unresponsiveness to H1-antihistamines, almost daily need for systemic corticosteroids and high UAS7, treatment with omalizumab was initiated. The therapy was started with $150 \mathrm{mg}$ subcutaneously

(s.c.) every 4 weeks for 3 months. There was no expected improvement after four doses and her UAS7 was 30-36, the dose was increased to $300 \mathrm{mg}$ s.c.

After two doses disease activity reduced and UAS7 was 14 . Simultaneously, further impairment in thyroid function tests was observed and levothyroxine therapy was started. Omalizumab therapy lasted for 12 months without side-effects and with significant reduction in disease activity and UAS7, and with improvement in overall quality of life of the patient and her family. 